Pulsars : Problems \& Progress

ASP Conference Series, Vol. 105,1996

S. Johnston, M. A. Walker and M. Bailes, eds.

\title{
Velocity-Magnetic Field Correlation of Pulsars
}

\author{
Naoki Itoh and Takemi Kotouda \\ Department of Physics, Sophia University, 7-1 Kioi-cho, Chiyoda-ku, \\ Tokyo, 102 Japan
}

\section{Introduction and Summary}

Monte Carlo simulations of the evolution of pulsars are carried out in order to compare with the recent measurement of the pulsar transverse velocity by Lyne \& Lorimer (1994). The new electron density distribution model of Taylor \& Cordes (1993) is adopted in the simulation. Accurate pulsar orbits in the Galactic gravitational field are calculated. It is found that the constant magnetic field model of pulsars can account for the new measurement of the pulsar transverse velocity and the apparent correlation between the strength of the magnetic field and the transverse velocity of the pulsars. The present finding confirms the validity of the constant magnetic field model of pulsars and consolidates the idea that the apparent correlation between the strength of the magnetic field and the transverse velocity of the pulsars is caused by observational selection effects.

\section{Simulation Results}

We show an example of the simulation. We adopt the following parameters: $\bar{P}_{0}$ $=10^{-1} \mathrm{~s}, \sigma_{u}=0.18, \bar{Q}_{0}=10^{-6.85} \mathrm{~s}^{\frac{1}{2}}, \sigma_{w}=0.45, \alpha_{i}=-2.28, \beta_{i}=\frac{2.28}{3}, \gamma_{i}=$ $11.64, \sigma_{\lambda}=0.8, \bar{s}=10^{2.60} \mathrm{~km} \mathrm{~s}^{-1}, \sigma_{s}=0.45 r_{b}=7.92 \times 10^{-3} \mathrm{yr}^{-1}$. The value of $\bar{Q}_{0}$ corresponds to $B=3.2 \times 10^{12} \mathrm{G}$. The Kolmogorov diagrams for $\log P$, $\log \dot{P}, \log L_{r}$, and $\log V_{t}$ are shown.

The correlation diagrams for the transverse velocity and the strength of the magnetic field are shown. The simulation automatically reproduces the observed correlation. The apparent correlation is caused by the lack of old high-velocity pulsars which are generally distant from us and difficult to observe.

\section{References}

Itoh, N., \& Hiraki, K. 1994, ApJ, 435, 784

Itoh, N., Kotouda, T., \& Hiraki, K. 1995, ApJ

Lyne, A. G., \& Lorimer, D. R. 1994, Nature, 369, 127

Taylor, J. H., \& Cordes, J. M. 1993, ApJ, 411, 674

Wakatsuki, S., Hikita, A., Sato, N., \& Itoh, N. 1992, ApJ, 392, 628 


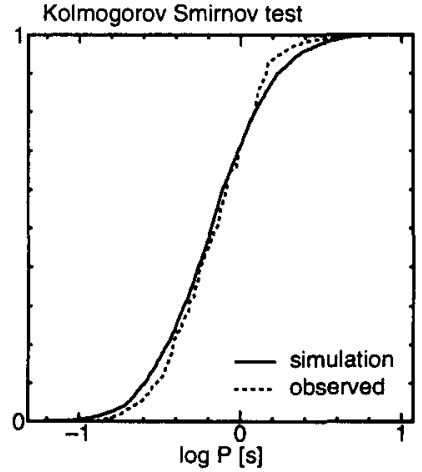

probability $=39.6 ! s$

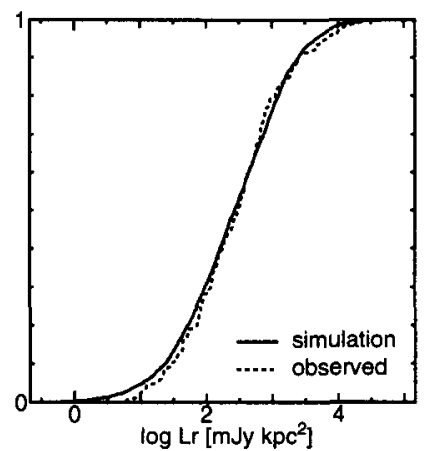

probability $=69.2 \mathrm{ss}$

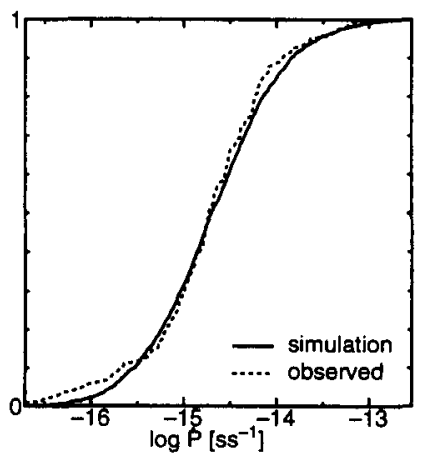

probability $=66.6 ! \mathrm{s}$

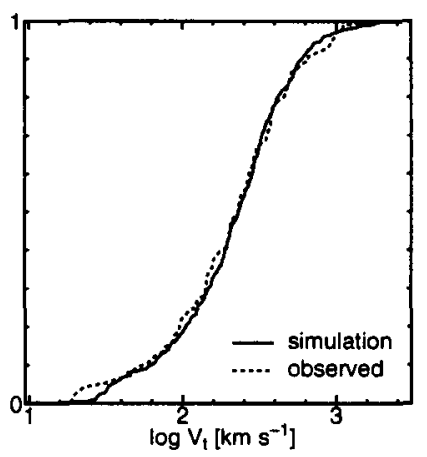

probability $=93.6 ! \mathrm{s}$
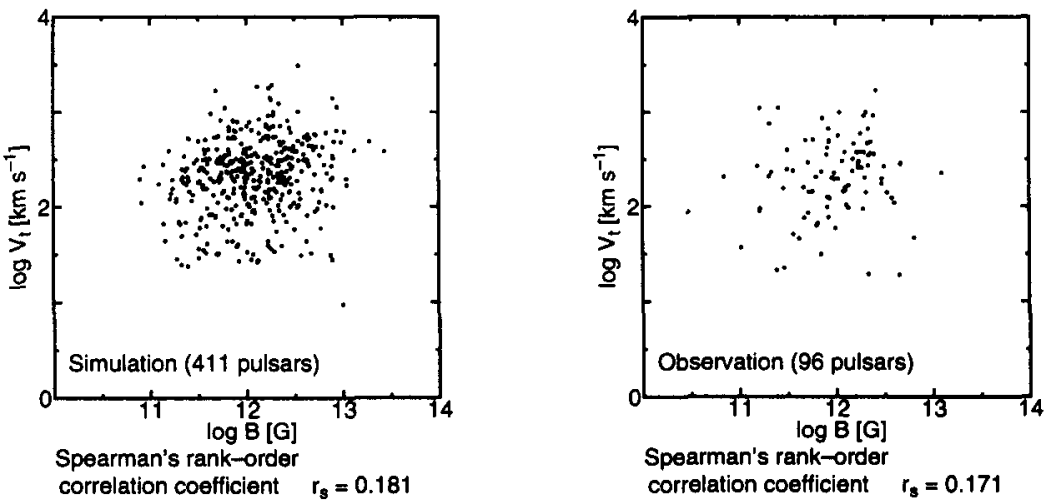